\title{
Assessment and management of patients with intestinal failure: a multidisciplinary approach
}

This article was published in the following Dove Press journal:

Clinical and Experimental Gastroenterology

\section{Jennie T Grainger' \\ Yasuko Maeda ${ }^{1,2}$ \\ Suzanne C Donnelly' \\ Carolynne J Vaizey ${ }^{1,2}$}

'The Lennard Jones Intestinal Failure Unit, St. Mark's Hospital, Harrow, UK; ${ }^{2}$ Faculty of Medicine, Imperial College London, London, UK
Correspondence: Yasuko Maeda St. Mark's Hospital, Northwick Park, Watford Road, Harrow HAI 3UJ, UK Tel +442082354164

Fax +442082354277

Email yazmaeda@gmail.com

\begin{abstract}
Intestinal failure (IF) is a condition characterized by the inability to maintain a state of adequate nutrition, or fluid and electrolyte balance due to an anatomical or a physiological disorder of the gastrointestinal system. IF can be an extremely debilitating condition, significantly affecting the quality of life of those affected. The surgical management of patients with acute and chronic IF requires a specialist team who has the expertise in terms of technical challenges and decision-making. A dedicated IF unit will have the expertise in patient selection for surgery, investigative workup and planning, operative risk assessment with relevant anesthetic expertise, and a multidisciplinary team with support such as nutritional expertise and interventional radiology. This article covers the details of IF management, including the classification of IF, etiology, prevention of IF, and initial management of IF, focusing on sepsis treatment and nutritional support. It also covers the surgical aspects of IF such as intestinal reconstruction, abdominal wall reconstruction, and intestinal transplantation.
\end{abstract}

Keywords: intestinal failure, short bowel syndrome, nutrition, abdominal wall reconstruction, multidisciplinary approach, enterocutaneous fistula

\section{Introduction}

Intestinal failure (IF) is a condition which is characterized by the inability to maintain a state of adequate nutrition or fluid and electrolyte balance due to an anatomical problem (i.e., inadequate length of small bowel), or a physiological disorder of the gastrointestinal (GI) system. ${ }^{1}$ IF can be an extremely debilitating condition, significantly affecting the quality of life of those affected, with a wide range of potential etiologies. It can be due to an acquired or congenital cause, GI or systemic, benign or malignant disease, and can affect any age, and the resulting state of malabsorption and malnutrition can vary in severity and duration.

The surgical management of patients with acute and chronic IF requires a specialist team who has the expertise in terms of technical challenges and decision-making. To produce the maximal outcome for the IF patient, the recent European Society of Coloproctology (ESCP) consensus statement ${ }^{1}$ states that IF should be a recognized sub-specialty within GI surgery to establish a surgical standard. A dedicated IF unit will have the expertise in patient selection for surgery, investigative workup and planning, operative risk assessment with relevant anesthetic expertise, and a multidisciplinary team with support such as nutritional expertise and interventional radiology. Clearly, the role of the surgeon is enhanced and complemented by those members of the multidisciplinary team to improve outcomes with reduction in morbidity and mortality, 
along with a beneficial effect of the cessation of dependence on parenteral nutrition (PN) postoperatively.

\section{Definition and classification of intestinal failure}

The term "intestinal failure" (IF) was defined originally by Fleming and Remington in 1981, to describe a state of "reduction in functioning gut mass below the minimal amount necessary for adequate digestion and absorption of food". ${ }^{2}$ The definition usually encompasses those patients who require PN to survive, but doesn't take into account those patients who, rather than needing full $\mathrm{PN}$, need regular electrolyte or fluid support. Recent work from The European Society for Clinical Nutrition and Metabolism (ESPEN) ${ }^{3}$ defines IF as "the reduction of gut function below the minimum necessary for the absorption of macronutrients and/or water and electrolytes, such that intravenous supplementation is required to maintain health and/or growth", which states IF occurs "when there is reduced intestinal absorption so that macronutrient and/or water and electrolyte supplements are needed to maintain health". ${ }^{4}$ ESPEN also described a state of "intestinal insufficiency" when a reduction in gut absorptive function doesn't require intravenous supplementation to maintain health and/or growth (or "intestinal deficiency" for those languages where "insufficiency" and "failure" have the same meaning).

\section{Functional classification}

IF can be classified according to its onset, metabolic, and expected outcome criteria into types 1,2 , and 3 . The classification depends upon the duration of nutritional support required and the reversibility of original pathology. ${ }^{1,5,6}$

- Type 1: This is a short-term, acute, and usually selflimiting condition. It usually refers to IF that lasts for a duration of less than 28 days and can be related to postoperative ileus or acute intestinal obstruction. It may require short-term $\mathrm{PN}$. Postoperative ileus is estimated to occur in about $10 \%-30 \%$ of patients in the perioperative setting,,$^{7,8}$ either after abdominal surgery, where significant bowel handling or concomitant sepsis has occurred. ERAS programs have been shown to reduce the incidence of post-operative ileus. ${ }^{9}$

- Type 2: This is a prolonged condition, considered to be a duration of greater than 28 days, and requires artificial nutrition. It tends to involve medically unstable patients who may have suffered septic, metabolic, and nutritional complications as a result of major bowel surgery. ${ }^{5}$ These patients require a multidisciplinary approach with metabolic and nutritional support for recovery pending spontaneous resolution or surgical treatment. Typical patients in this group include those with complex Crohn's disease, intestinal fistulas, or abdominal sepsis.

- Type 3: This is a chronic IF that requires long-term parenteral support. Type 3 may be irreversible and occurs as a consequence of short bowel syndrome, either due to loss of length of small bowel from a massive small bowel resection or due to a loss of absorptive capacity of the small bowel. ${ }^{10}$ The etiology for type 3 IF in the UK has changed over the years, with surgical complications now accounting for most cases $(28.8 \%)$, followed by inflammatory bowel disease $(22.6 \%) .{ }^{5,11}$ Malignancy or radiation enteritis carries a poorer prognosis, with a survival rate of $62 \%$ at 1 year. $^{11}$

Patients with type 1 and the majority of those with type 2 IF would be expected to return to full intestinal autonomy with time. Type 3 IF involves patients with established short bowel syndrome who require long-term PN.

From here on, the main focus will be on those patients who have type 2 IF and their management.

\section{Etiology}

The most common cause of type 2 IF is secondary to a complication of abdominal surgery, such as an anastomotic leak or intestinal injury at the time of surgery, with postoperative complications ranging from $32 \%$ to $50 \% .^{2,5,12-14}$ Abdominal catastrophe such as intestinal ischemia, trauma, or a volvulus which requires a significant small bowel resection, with or without the colon, are also common causes. ${ }^{5}$

The exact incidence or prevalence of type 2 IF is difficult to quantify. It is estimated that, in England, there are between 1,500 and 2,000 inpatient stays per year. ${ }^{15}$

\section{Prevention of intestinal failure}

One of the commonest causes of type 2 IF is surgical complications; patient selection, timing of surgery, and surgical technique all have an impact on prevention of type 2 IF. Minimizing the risk factors prior to surgery, which include controlling sepsis, correcting hypoalbuminemia, controlling immunosuppression, and draining preexisting intraabdominal collections, would all help to reduce the incidence of anastomotic leaks and sepsis.

It is important to optimize the nutritional status of these patients preoperatively. Malnourished patients are at higher risk of postoperative morbidity and mortality than 
well-nourished patients undergoing similar operations. ${ }^{16}$ The role of nutrition support in the management of enterocutaneous fistulas is primarily one of supportive care to prevent malnutrition, thereby obviating further deterioration of an already debilitated patient. It has been shown in several studies that PN has substantially improved the prognosis of GI fistula patients by increasing the rate of spontaneous closure and improving the nutritional status of patients requiring repeat operations. Moreover, other studies have shown that nutritional support decreases or modifies the composition of the GI tract secretions and is, thus, considered to have a primary therapeutic role in the management of fistula patients. ${ }^{17}$

Surgical techniques, including careful dissection and close inspection of all the bowel to identify any inadvertent enterotomy, on table air tests and decision-making regarding whether to anastomose, are all important in avoiding postoperative catastrophe.

\section{Initial management of intestinal failure}

Distinguishing type 1 and type 2 IF relates to the duration of illness and its severity. Initially, it may not be obvious who is type 1 versus type 2 , and they may become a continuum of the same condition. ${ }^{5}$

If a patient has absent GI function after 5 days, a decision is required if further investigation is required at this stage. This will depend on the overall status of the patient and may include a computed tomography (CT) scan to rule out intraabdominal sepsis or a leak.

Reversible metabolic factors that could be contributing to poor gut function should be looked for and reversed, e.g., abnormal electrolytes. Together with clinical assessment, the underlying cause for the absent GI function and the likely chance of resolution should be taken into account. Early involvement of the nutritional team with these patients is beneficial. If the illness is likely to be ongoing at this stage, nutritional support should be considered. PN is generally indicated if the patient has failed, or is expected to fail, to tolerate an adequate enteral diet for 5-7 days. ${ }^{5}$

Sepsis control is important in the management of the acute phase of IF, especially type 2 . Patients need to be resuscitated and managed according to the sepsis bundle. ${ }^{18}$ Source control is a priority, and cross-sectional imaging may be required to identify intra-abdominal collections, which ideally should be controlled by percutaneous drainage as the treatment of choice. ${ }^{19}$ With the increase in availability of cross-sectional imaging and interventional radiology, advances have been made in the diagnosis and nonoperative control of abdominal and pelvic collections, and the vast majority of patients can be controlled without early open surgery. ${ }^{20}$

Radiological drainage may not be possible if collections are inaccessible (e.g., multiple collections between bowel loops), or not appropriate if there is an anastomotic leak with peritonitis. In these cases, an early return to theatre may be needed. If it is more than 10 days after the original laparotomy, the peritoneal cavity is hostile and can result in further injury to the patient. The minimum amount of surgery should be undertaken to enable adequate drainage of infection, resection of any perforation, and then formation of stomas. In this situation, any enterotomies or resection areas should not be anastomosed or sutured, as the risk of complication is high. Enterotomies should be exteriorized or, if repaired, should be defunctioned proximally. ${ }^{5}$

Antibiotics are an important adjunct in the treatment of sepsis. This may need discussion with microbiology, as often these patients have had multiple courses of antibiotics previously, and it is important to determine the most suitable antibiotics and duration to avoid these patients developing resistance. Rapid commencement in these patients once sepsis is confirmed is needed as there is good evidence to show a strong relationship between the timeliness of therapy and survival. ${ }^{20}$

Once acute sepsis and urgent conditions requiring surgical intervention were excluded and immediate nutrition addressed to stabilize the patient, further workup for reconstructive surgery becomes key. For type 2 IF, there is a clear sequence to manage these patients.

The "sepsis-nutrition-anatomy-plan" approach has always been the therapeutic strategy for managing patients with $\mathrm{IF}^{2,21}$ More recently, ESPEN have modified this to state that the key aspects are sepsis control, fluid and electrolyte resuscitation, optimization of nutritional status, wound care, appropriate surgery, and active rehabilitation. ${ }^{22}$

\section{Sepsis}

Adequate management of abdominal sepsis is a key factor in determining the outcomes in patients with acute $\mathrm{IF}^{23}$ Once the patient has entered the "stable" phase of IF, the onset of infection may be less obvious and insidious, with no evidence of temperatures or rise in white cell count, and instead a chronic infection may manifest with difficulty in gaining weight, hypoalbuminemia, hyponatremia, and even jaundice. ${ }^{24}$ Throughout their disease, a high index of suspicion for the presence of sepsis should be maintained. 
The detrimental effect of sepsis on patients with IF is multifactorial. Spontaneous healing of a fistula is less likely in the presence of active infection. ${ }^{25}$ Resolution of sepsis is fundamental for maintaining adequate nutrition, as infection is associated with an increased metabolic demand and impaired fuel utilization. Septic patients will continue to be in a catabolic state and lose proteins and calories despite PN. This leads to progressive weight loss, meaning that aggressive nutritional support is less likely to be effective until the sepsis is treated. ${ }^{2,26}$

\section{Nutritional support}

Patients who are not nutritionally stable will suffer difficulties with wound healing due to a lack of both macronutrients and micronutrients, ${ }^{27,28}$ and will also have an impaired immune response. ${ }^{2}$ Regular anthropometry, nutritional bloods, and accurate adequate fluid balance are vital in these patients.

Nutrition should be tailored to the individual patients by route and quantity. Enteral feeding is the route of choice in all patients with a functioning GI tract. This may be limited in type 2 IF due to the presence of inflammation, strictures, obstruction, radiation damage, short bowel, and intestinal fistulas. Enteral feeding can not only reduce the complications from PN, but it is also important for the psychological health of these patients.

In patients with a high stoma output, especially from a short bowel, distal feeding may be of nutritional benefit, if it is accessible. An additional theoretical benefit of distal feeding into small bowel is the maintenance of its function and its caliber in anticipation of a future anastomosis. Distal feeding, done by placing a gastrostomy feeding tube into the intestine distal to the fistula, can replace the need for parenteral feeding in selected patients. ${ }^{29}$ We tend to use oral nutritional supplements in this case. This may be suitable for patients with an open abdomen, and is cheap and safe. If it is done only to maintain bowel health and caliber, bolus feeding with saline may be sufficient. Stoma nurses need to be on board, as the stoma bag needs to be changed to have a window for access, and nutrition nurses need to cannulate the bowel and train the patient to do this as well.

Once it is clear that a fistula is established, there is no evidence that oral intake will prevent healing. Eating is psychologically beneficial, and frequent meals can enhance the number of calories absorbed.

The amount and type of fluid that the patient is allowed to drink depends on the position of the fistula or stoma. If the fistula or stoma is of low output and is known to be in the distal small bowel or colon, this can be managed like a standard ileostomy. The patient can, therefore, eat and drink with standard dietary advice for an ileostomy. If the fistula or stoma is high, the fluid intake may have to be modified with restriction of hypotonic fluids to $500-1000 \mathrm{~mL}$ per day, and the addition of an electrolyte fluid, such as St Mark's electrolyte mix, double strength Dioralyte (2 sachets in 200 $\mathrm{mL}$ water five times per day or 10 sachets in $1 \mathrm{~L}$ of water) or Glucodrate. Some people need much more strict fluid restriction. Hypotonic fluids (e.g., WHO solution) draw salt, and with it water, into the gut which increases the fistula or stoma outputs significantly. Diet drinks and food containing polyols should also be avoided.

PN tends to be the mainstay of nutritional support for type 2 IF. Complications associated with long-term PN include line complications and liver disease. Improvements in catheter care such as use of single lumen and dedicated tunneled central venous catheters for nutrition have led to a reduction in PN-associated infective complications. ${ }^{2}$ Liver disease risk associated with long-term PN increases as the duration of PN increases and is the main cause of death in those patients with permanent IF. ${ }^{30}$

In reality, patients may need a combination of both enteral and PN depending on the degree of dysfunction of their GI tract. What is important is that they receive adequate nutrition to meet their metabolic needs to build them up and prepare them for reconstructive surgery. This process can take several months, preferably with nutritional support at home, where patients can eat nonhospital food, mobilize more, feel more psychologically normal, and are away from hospital-acquired infections.

\section{Wound and fistula care}

Wound, fistula, and stoma care can be complex for these patients and require specialist stoma therapy nurses. These patients should be managed on a ward where the staff have sufficient expertise and resources to be able to manage all wound issues competently, especially out of hours. The inability to get control of the wound or fistula can be a source of morbidity for the patient, as they experience pain and severe discomfort from the skin when leakages occur. Difficulty with management of these complex wounds may necessitate transfer to a specialist unit. ${ }^{5}$ The presence of an open abdomen with a fistula is particularly difficult to manage. These wounds may be best managed with a large wound care (Eakin) bag, with topical suction catheters placed through the bag to control the effluent and protect the skin. ${ }^{31}$ 


\section{Radiology}

In the acute setting, initial radiological assessment is required to exclude and treat abdominal sepsis. Early cross-sectional imaging is optimal for this and can also be used to look for portal hypertension - a condition that may need treating before major abdominal surgery.

The next stage is to define the proximal and distal gut lengths and integrity with a view to planning oral and distal feeding. Radiological assessment is also needed to identify any potential reason why a fistula may not have healed in the first place. Residual disease such as inflammation and stricturing may be revealed.

Prior to embarking on any surgery, it is advisable to review the entire gut radiologically, with the addition of endoscopy where required. It is essential to exclude distal narrowing prior to any anastomosis. Water-soluble iodinated contrast is preferred to barium if perforation or a leak is suspected, as the extravasated barium can cause an inflammatory response in the peritoneum. Barium does, however, have a greater sensitivity for demonstrating enteric fistulae. ${ }^{32,33} \mathrm{In}$ some cases, direct visualization of the mucosa is required with endoscopy. This can help to assess the pathology underlying strictures or inflammation, which may be difficult to fully distinguish on radiological imaging alone.

The amount of remaining bowel, together with the patient's projected activity levels, helps to determine the likelihood that patients will require PN following the surgery. This allows for an informed discussion with the patient about what surgery will entail and the risk of the requirement for PN postoperatively. If several fistulae are present, consideration to the timing of investigations is important to avoid confusion. Often a contrast study proximal to the fistula opening is examined first, otherwise defunctioned bowel containing contrast can degrade the image quality and lead to confusion of the anatomy. ${ }^{34}$

In those patients with Crohn's, anal sepsis should be excluded, and this may require an MRI done on a fistula protocol or even an examination under anesthesia. In patients with an ileal conduit or those with other complex past urological surgery, the anatomy of the urinary tract should be established prior to surgery. The need for preoperative ureteral stenting should also be considered. Patients who have suffered extensive mesenteric ischemia and who have severe cardiovascular disease should be assessed preoperatively with a CT angiography to rule out insufficient blood supply to the gut. ${ }^{1}$ Other imaging, such as fistulography, vaginography, or cholangiography, may also help to build the anatomical picture.
CT scanning of the abdomen will help to determine the size of any abdominal wall defect to aid surgical planning in terms of operating time, the requirement of a mesh, or even the need to have a plastic surgeon as part of the operating team. ${ }^{33,35}$ Scrolling up and down the midline on the images will provide information on the optimal entry site into the abdomen.

These patients can have a complex anatomy that is difficult to define and establish. An experienced radiologist used to dealing in complex GI radiology is required to assess and review any imaging. Involving the radiologist early in the course can be beneficial with regard to radiology planning and choice of investigations. Often, all imaging will be reviewed in an multidisciplinary team setting with the surgeons, radiologists, and gastroenterologists before definitive surgery is planned, and a designated radiologist would be responsible for this complex cohort of patients.

\section{Pain control}

Many patients with IF become dependent on large doses of analgesics, especially as the oral access for analgesia may be impaired.

Chronic use of opioid and non-opioid analgesics is common in many patients with IF, especially in those with enterocutaneous fistula. Oral analgesia may not be appropriate in patients with a very short gut or obstruction. Intravenous access may also be difficult. The use of patches can provide more sustained drug levels.

Early involvement of a pain team with an understanding of the effects of analgesics on gut function, access to nonpharmacological distraction techniques, and a psychiatrist or psychologist with an understanding of addiction can be extremely helpful.

It is also important to reduce analgesic requirements prior to surgical intervention, or postoperative pain control can be extremely difficult, with little scope for escalation of pain management if needed.

The management of pain after major open abdominal surgery relies on two techniques: patient-controlled analgesia using an opioid self-administered in small doses by the patient and epidural analgesia. Ideally, analgesic regimes should take into account periods where pain intensity is increased due to therapeutic interventions, e.g., physiotherapy, and dressing changes.

\section{Intestinal reconstruction}

Timing of surgery is an important consideration for IF patients. In a complex or open abdomen, it is thought that 
the process of forming a new peritoneal cavity takes around 6 months. ${ }^{36}$ The best indicator for when the abdomen has matured for surgery is when prolapse of the bowel is evident at the point of a fistula. With experience, evaluation of whether an abdomen has "matured" can be done by palpation of the abdomen. Up to this point, the abdomen is still extremely hostile, and surgery puts the patient at risk of further injury and complications.

The advantage of bringing the distal bowel into continuity has to be weighed against the risk of an anastomotic leak. Surgery for type 2 IF is usually life changing, rather than life saving in the short term, and patients have to be counseled appropriately regarding this. In the long term, the advantages may include reduction or cessation of $\mathrm{PN}$ with avoidance of the associated risks.

The surgery itself should be allocated plenty of time. A second surgeon should be considered in long and complex cases, so that fatigue and reduction in concentration don't put the patient at risk of injury. Careful dissection, with adequate time examining the bowel prior to closure, should be performed to reduce the risk of an enterotomy. Every serosal tear should be repaired, and any anastomosis should be performed meticulously. The anastomosis should not be placed within an abscess cavity, nor near an incision. A hand-sewn, 2-layer anastomosis reduces the risk of recurrence of fistula marginally when compared with a stapled anastomosis. ${ }^{37}$ If a stapled anastomosis is to be used, it is recommended that all staple lines are oversewn. ${ }^{1}$ Often the distal bowel can be atrophic and of small caliber. In these cases, a proximal defunctioning stoma may be worth considering. Patients should be routinely booked into a high dependency unit postoperatively, and adequate postoperative analgesia prescribed.

\section{Abdominal wall reconstruction}

Abdominal wall reconstruction may be a vital component of these operations, especially if the patient has had a previous laparostomy. Failure to gain closure of the abdominal wall properly may lead to re-fistulation, formation of a large incisional hernia, and poor cosmetic result. ${ }^{1}$ The simultaneous resection of the GI tract with the abdominal wall increases the morbidity of surgery. These operations should, therefore, be performed in a specialist center, ${ }^{1}$ where the surgical and anesthetic teams are used to dealing with such complex cases and any complications that could arise.

The use of mesh may be required due to the presence of multiple defects, a large midline defect with poor rectus bulk, or a history of previous component separation. The evidence for the safety and efficacy of nonabsorbable mesh in the open abdomen with enteric fistulization is limited, but it is thought to be associated with increased infection rates. ${ }^{38-40}$ If infection develops with a nonabsorbable mesh, removal is often required necessitating a further operation. There is evidence that simultaneous fistula surgery and abdominal wall reconstruction should not be performed with a non-absorbable mesh or cross-linked collagen mesh. The initial comparative study reported $0 \%$ re-fistulization in patients reconstructed with sutures alone, $24 \%$ when a nonabsorbable mesh was used, and $41.7 \%$ when a cross-linked collagen mesh was used. ${ }^{41,42}$ Two studies looking at the use of a non-cross-linked collagen mesh in this setting reported re-fistulation rates of $9 \%-11 \%{ }^{43,44}$ Therefore, if primary closure employing a sutured repair is possible, this is the safest option. Techniques employing autologous tissue such as component separation and primary repair are recommended..$^{45-47}$

Nonabsorbable synthetic meshes and cross-linked biological implants should not be placed into the peritoneal cavity in IF due to the reported incidence of increased complications, with rates of re-fistulization between $22 \%$ and $25 \%$ with these techniques. ${ }^{48,49}$ The use of a nonabsorbable synthetic mesh should be limited to an onlay repair and should never be placed within the peritoneal cavity. They should be avoided in a contaminated environment. ${ }^{1}$

If it is not possible to bring together the abdominal wall, even with a component separation, or if a mesh is required in a contaminated field, a non-cross-linked biological mesh should be used. Most biological meshes used outside the abdominal cavity lead to seroma formation, and drains should be left in the abdominal wall until dry, even if this amounts to several weeks. ${ }^{50}$ Larger defects, in both the abdominal wall and the skin, may require a plastic surgeon to perform a reconstruction using autologous sliding or pedicled flaps. ${ }^{1}$

\section{Intestinal transplantation}

PN is the preferred treatment option for patients with short bowel syndrome and chronic IF because of its superior survival outcome (63\% at 5 years compared with $54 \%$ in intestinal transplantation). ${ }^{13,51}$ However, long-term PN can fail due to loss of venous access from catheter-associated thrombosis, recurrent catheter-related blood stream infections, and liver dysfunction.

Intestinal transplantation offers a rescue therapy for chronic IF patients, with indications for transplant falling into four categories:

- Impending or overt liver failure due to PN-induced liver injury; 
- Thrombosis of two or more major central veins;

- Frequent life-threatening central venous catheter infections and sepsis. A single episode of central line fungaemia, septic shock, or ARDS is considered failure of PN, but must be reviewed by the PN center before referral for transplant; and

- Frequent severe episodes of dehydration, despite fluid replacement and $\mathrm{PN}$.

A simulation study from the US comparing both home parenteral nutrition and intestinal transplantation for irreversible IF has shown that intestinal transplant marginally improved survival in those patients who had intestinal transplantation (14.6 years vs 14.9 years) when offered to patients on long-term PN with a life expectancy of $<12$ months if they do not undergo surgery. ${ }^{52}$

Three types of intestinal transplantation are currently available and include isolated small bowel, liver plus small bowel, and multi-visceral. The current 5-year survival rate is $54 \%$, with an isolated small bowel transplant, which is worse than the outcome associated with PN (63\% at 5 years). ${ }^{13,51}$ Outcomes are improving due to the standardization of surgical techniques, advances in immunosuppressive therapy, and improvements in postoperative patient care, but continue to carry a risk of complications (such as rejection and infections) and adverse events associated with immunosuppression. ${ }^{53-55}$ These patients often require frequent hospital admissions or blood tests due to complications of their immunosuppressive regimes. Some patients continue to have problems of "dependency". Although the patient is relieved of PN dependence, they endure close monitoring of their immunosuppression, more septic episodes, prolonged hospital stays, and often significant limitations on their personal life as a result. ${ }^{56}$

Consideration for intestinal transplantation should be discussed at an IF network meeting, ${ }^{1}$ with the IF unit caring for the patient and the transplant team, and primary management of these patients should be provided by a center experienced in medical intestinal rehabilitation, nutrition, and transplantation of other solid organs.

\section{Multidisciplinary team approach}

Patients with type 2 IF are complex and time consuming, and have a protracted hospital episode. Regardless of the etiology, the management requires a considerable length of hospital stay and extensive multidisciplinary input. Dieticians, stoma therapists, nurses, microbiologists, pharmacist, radiologists, physiotherapists, anesthetists, pain specialists, psychiatrists, nutrition nurses, gastroenterologists, physicians, and surgeons all act together to provide an appropriate input to the patients care throughout their hospital admission, their outpatient rehabilitation, and in the buildup to their definitive reconstructive surgery.

Restorative surgery should be deferred where possible until the patient is nutritionally stable, mobilizing, and medically and psychologically optimized. In the preoperative phase, patients should be given the opportunity to meet with an anesthetist to address any comorbid issues. Issues regarding postoperative pain relief, nutritional support, and intravenous (IV) access should also be addressed. The gastroenterology team is crucial in the preoperative workup of all IF patients, but especially those with underlying inflammatory bowel disease for control, or correction of their underlying condition and postoperative disease management. In patients with a fistula, other specialties, such as urology, gynecology, and plastic surgery, may also be involved.

This multidisciplinary team approach is also vital in the postoperative phase of the patient management. ${ }^{5}$ An active program of mobilization and psychological support is important. The majority of IF patients will have depleted lean muscle mass. Therefore, an active, graded mobilization program is needed to help expedite recovery. ${ }^{5}$ Many patients with type 2 IF will require psychological support. The patient and their family often have anxiety, a feeling of loss, loss of self-esteem, depression, and anger, particularly if they developed type 2 IF secondary to a postoperative complication. They may develop body image issues related to stomas, fistulae, and an open abdomen. IF can also have huge financial implications, as the patient suffers a loss of earnings and financial hardship. ${ }^{36}$ These issues can inhibit their recovery and should be addressed early on. This may prevent the impact of further psychological complications later. It is also important to provide explanations to the patient at each stage of their recovery, as a number of them may have spent prolonged periods in hospital and had numerous investigations and invasive interventions, without fully understanding the reason for each step. Patients are often frustrated, do not understand the benefit of waiting to get conditions optimal prior to surgery, and should participate in discussions surrounding their care.

The ward nursing staff, specialist nutrition nurses, and the nutrition support team are majorly involved in the delivery of care to these patients. The nutrition support team are an essential part for any patient, and usually will act to facilitate the transition of patients care from in-patient to out-patient. ${ }^{5}$ Patients with these conditions need to be managed in a designated unit that has the sufficient expertise, interest, and volume of patients to improve the outcome for patients t, $^{1,5,37,57}$ 
and a multidisciplinary and multimodal approach is likely to increase survival in these complex patients. ${ }^{58}$

If possible, patients with type 2 IF should be discharged home prior to their surgery, ${ }^{1}$ provided that home PN, complex wound care, supervision of home care, and regular monitoring of out-patients are available.

\section{Disclosure}

The authors report no conflicts of interest in this work.

\section{References}

1. Vaizey CJ, Maeda Y, Barbosa E, et al. European Society of Coloproctology consensus on the surgical management of intestinal failure in adults. Colorectal Dis. 2016;18(6):535-548.

2. Fleming CR, Remington M. Intestinal failure. In: Hill GL, editor. Nutrition and the Surgical Patient. Edinburgh: Churchill Livingstone; 1981:219-235.

3. Pironi L, Arends J, Baxter J, et al. ESPEN endorsed recommendations. Definition and classification of intestinal failure in adults. Clin Nutr. 2015;34(2):171-180.

4. Nightingale JMD. Definition and classification of intestinal failure. In: Nightingale JMD, editor. Intestinal Failure. London: Greenwich Medical Media; 2001:xix-xx.

5. Carlson G, Gardiner K, McKee R, MacFie J, Vaizey C; The Surgical Intestinal Failure Working Party on behalf of the Association of Surgeons of Great Britain and Ireland. The Surgical Management of Patients with Acute Intestinal Failure. London: Association of Surgeons of Great Britain and Ireland; 2010.

6. Lal S, Teubner A, Shaffer JL. Review article: intestinal failure. Aliment Pharmacol Ther. 2006;24(1):19-31.

7. Venara A, Neunlist M, Slim K, et al. Postoperative ileus: pathophysiology, incidence and prevention. J Visc Surg. 2016;153(6):439-446.

8. Wolff BG, Viscusi ER, Delaney CP, Du W, Techner L. Patterns of gastrointestinal recovery after bowel resection and total abdominal hysterectomy; pooled results from the placebo arms of alvimopan phase III North American clinical trials. J Am Coll Surg. 2007;(1)205:43-51.

9. Barbieux J, Hamy A, Talbot MF, et al. Does enhanced recovery reduce postoperative ileus after colorectal surgery? J Visc Surg. 2017;154(2):79-85.

10. Elia M, Smith RM, on behalf of BAPEN and its collaborators. Improving Nutritional Care and Treatment. Perspectives and Recommendations from Population Groups, Patients and Carers. Redditch: BAPEN; 2009.

11. Oke S, Lloyd D, Nightingale J, Gabe S. AODWE-009 Changes in aetiology of type 3 intestinal failure over 36 years: a single centre experience. Gut. 2017;66(Suppl 2):A124-A125.

12. Carlson G, Dark P. Acute intestinal failure. Curr Opin Crit Care. 2010;16(4):347-352.

13. Oke S, Lloyd D, Nightingale J, Gabe S. OC-022 Factors that affect survival in type 3 intestinal failure; the largest single centre experience of 978 patients over 36 years. Gut. 2017;66(Suppl 2):A11-A12.

14. Scott NA, Leinhardt DJ, O'Hanrahan T, Finnegan S, Shaffer JL, Irving MH. Spectrum of intestinal failure in a specialised unit. Lancet. 1991;337(8739):471-473.

15. NHS England. Available from: https://www.england.nhs.uk/wp-content/ uploads/2013/06/a08-intestinal-failure-adult.pdf. Accessed May 25, 2018.

16. Dempsey DT, Mullen JL, Buzby GP. The link between nutritional status and clinical outcome: can nutritional intervention modify it? Am J Clin Nutr. 1988;47(Suppl 2):352-356.

17. Dudrick SJ, Maharaj AR, McKelvey AA. Artificial nutritional support in patients with gastrointestinal fistulas. World J Surg. 1999;23(6): $570-576$.
18. Howell MD, Davis AM. Management of sepsis and septic shock. JAMA. 2017;317(8):847-848.

19. Shirah GR, O’Neill PJ. Intra abdominal infections. Surg Clin North Am. 2014;94(6):1319-1333.

20. Soop, M, Carlson GL. Recent developments in the surgical management of complex intra-abdominal infection. Br J Surg. 2017;104(2): e65-e74.

21. Williams NM, Scott NA, Irving MH. Successful management of external duodenal fistula in a specialised unit. Am J Surg. 1997;173(3): 240-241.

22. Klek S, Forbes A, Gabe S, et al. Management of acute intestinal failure: a position paper from the European society for clinical nutrition and metabolism (ESPEN) special interest group. Clin Nutr. 2016;35(6):1209-1218.

23. Carlson GL. Surgical management of intestinal failure. Proc Nutr Soc. 2003;62:711-718

24. Carlson GL and Irving MH. Infection: recognition and management of infection in surgical patients. In: Hanson GC, ed. Critical Care of the Surgical Patient: Companion Series to Bailey and Love's Short Practice of Surgery: Chapman \& Hall; 1997:273-290.

25. Rolandelli R, Roslyn JJ. Surgical management and treatment of sepsis associated with gastrointestinal fistulas. Surg Clin, North Am. 1996;76:1111-1122.

26. Carlson GL. Surgical causes and management of acute intestinal failure. In: Nightingale JM, editor. Intestinal Failure. London: Greenwich Medical Media; 2001:41-49.

27. Aksamija, G, Mulabdic A, Rasic I, Aksamija L. Evaluation of risk factors of surgical wound dehiscence in adults after laparotomy. Med Arch. 2016;70(5);369-372.

28. Williams JZ, Barbul A. Nutrition and wound healing. Surg Clin North Am. 2003;83:571-596

29. Teubner A, Morrison K, Ravishankar HR, Anderson ID, Scott NA, Carlson GL. Fistuloclysis can successfully replace parenteral feeding in the nutritional support of patients with enterocutaneous fistula. $\mathrm{Br} J$ Surg. 2004;91(5):625-631.

30. Cavicchi M, Beau P, Crenn P, Degott C, Messing B. Prevalence of liver disease and contributing factors in patients receiving home parenteral nutrition for permanent intestinal failure. Ann Intern Med. 2000;132(7):525-532.

31. Gardiner KR. Management of acute intestinal failure. Proc Nutr Soc. 2011;70(3):321-328.

32. Kwon SH, Oh JH, Kim HJ, Park SJ, Park HC. Interventional management of gastrointestinal fistulas. Korean J Radiol. 2008;9(6):541-549.

33. Thomas HA. Radiological investigation and treatment of gastrointestinal fistulas. Surg Clin North Am. 1996;76:1081-1094.

34. Kaushal M, Carlson GL. Management of enterocutaneous fistulas. Clin Colon Rectal Surg. 2004;17(2):79-88.

35. Pickhardt PJ, Bhalla S, Balfe DM. Acquired gastrointestinal fistulas: classification, etiologies and imaging evaluation. Radiology. 2002;224(1):9-23.

36. Joyce MR, Dietz DW. Management of complex gastrointestinal fistula. Curr Probl Surg. 2009;46(5):384-430.

37. Datta V, Engledow A, Chan S, Forbes A, Cohen CR, Windsor A. The management of enterocutaneous fistula in a regional unit in the United Kingdom: a prospective study. Dis Colon Rectum. 2010;53(2):192-199.

38. Carbonell AM, Criss CN, Cobb WS, Novitsky YW, Rosen MJ. Outcomes of synthetic mesh in contaminated ventral hernia repairs. $J$ Am Coll Surg. 2013;217(6):991-998.

39. Kurmann A, Barnetta C, Candinas D, Beldi G. Implantation of prophylactic nonabsorbable intraperitoneal mesh in patients with peritonitis is safe and feasible. World J Surg. 2013;37(7):1656-1660.

40. Slater NJ, Bokkerink WJ, Konijn V, Bleichrodt RP, van Goor H. Safety and duration of one-stage repair of abdominal wall defects with enteric fistulas. Ann Surg. 2015;261(3):553-557.

41. Connolly PT, Teubner A, Lees NP, Anderson ID, Scott NA, Carlson GA. Outcome of reconstructive surgery for intestinal fistula in the open abdomen. Ann Surg. 2008;247(3):440-444. 
42. Krpata DM, Stein SL, Eston M, et al Outcomes of simultaneous large complex abdominal wall reconstruction and enterocutaneous fistula takedown. Am J Surg. 2013;205:354-358; discussion 358-359.

43. Atema JJ, Furnee EJ, Maeda Y, et al. Major complex abdominal wall repair in contaminated fields with use of a non-cross-linked biologic mesh: a dual-institutional experience. World J Surg. 2017;41(8): 1993-1999.

44. Hodgkinson JD, Maeda Y, Leo CA, Warsavitarne J, Vaizey CJ. Complex abdominal wall reconstruction in the setting of active infection and contamination: a systematic review of hernia and fistula recurrence rates. Colorectal Dis. 2017;19(4):319-330.

45. Anderson O, Putnis A, Bhardwaj R, et al. Short- and long-term outcome of laparostomy following intra-abdominal sepsis. Colorectal Dis. 2011;13(2):e20-e32.

46. Petro CC, Como JJ, Yee S, Prabhu AS, Novitsky YW, Rosen MJ. Posterior component separation and transversus abdominus muscle release for complex incisional hernia repair in patients with a history of an open abdomen. J Trauma Acute Care Surg. 2015;78(2):422-429.

47. Wind J, van Koperen PJ, Slors JF, Bemelman WA. Single stage closure of enterocutaneous fistula and stomas in the presence of large abdominal wall defects using the components separation technique. Am J Surg. 2009;197(1):24-29.

48. Rosen MJ, Krpata DM, Ermlich B, Blatnik JA. A 5-year clinical experience with single-staged repairs of infected and contaminated abdominal wall defects utilizing biologic mesh. Ann Surg. 2013;257(6):991-996.

49. Shah BC, Tiwari MM, Goede MR, et al. Not all biologics are equal! Hernia. 2011;15(2):165-171.
50. Taner T, Cima RR, Larson DW, Dozois EJ, Pemberton JH, Wolff BG. Surgical treatment of complex enterocutaneous fistulas in IBD patients using human acellular dermal matrix. Inflamm Bowel Dis. 2009;15(8):1208-1212.

51. Drastich P, Oliverius M. Crohn's disease and intestinal transplantation. Dig Dis. 2017;35:127-133.

52. Roskott AM, Groen H, Rings EH, et al. Cost effectiveness of intestinal transplantation for adult patients with intestinal failure: a simulation study. Am J Clin Nutr. 2015;101(1):79-86.

53. Fishbein TM. Intestinal transplantation. N Engl J Med. 2009;361(10): 998-1008.

54. Mangus RS, Tector AJ, Kubal CA, Fridell JA, Vianna RM. Multivisceral transplantation: expanding indications and improving outcomes. J Gastrointest Surg. 2013;17(1):179-86; discussion:186-187.

55. Palmo A, DeFrancesco A, Costantino AM, et al. Potential adult candidates for intestinal transplantation; data from a single institutional experience during a 15-year period. Transplant Proc. 2002;34(3): 859-862.

56. Canovai E, Ceulemans L, Peers G, et al. Intestinal transplantation is less expensive compared to long-term parenteral nutrition in adults. Transplantation. 2017;101(6S2):S65.

57. Saalwachter AR, Evans HL, Willcutts KF, et al. A nutrition support team led by general surgeons decreases inappropriate use of total parenteral nutrition on a surgical service. Am Surg. 2004;70:1107-1111.

58. Corcos O, Castier Y, Sibert A, et al. Effects of multimodal management strategy for acute mesenteric ischaemia on survival and intestinal failure. Clin Gastroeneterol Hepatol. 2013;11:158-165e52.
Clinical and Experimental Gastroenterology

\section{Publish your work in this journal}

Clinical and Experimental Gastroenterology is an international, peerreviewed, open access, online journal publishing original research, reports, editorials, reviews and commentaries on all aspects of gastroenterology in the clinic and laboratory. This journal is included on PubMed. The manuscript management system is completely online

\section{Dovepress}

and includes a very quick and fair peer-review system, which is all easy to use. Visit http://www.dovepress.com/testimonials.php to read real quotes from published authors. 